\title{
Salinomycin Induces Reactive Oxygen Species and Apoptosis in Aggressive Breast Cancer Cells as Mediated with Regulation of Autophagy
}

\author{
KWANG-YOUN KIM ${ }^{1 *}$, KWANG IL PARK ${ }^{2 *}$, SANG-HUN KIM ${ }^{3}$, SUN-NYOUNG YU ${ }^{3}$, DEOKJAE LEE ${ }^{4}$, \\ YOUNG WOO KIM ${ }^{1}, \mathrm{KYUNG}$ TAE NOH ${ }^{5}$, JIN YEUL MA ${ }^{2}$, YOUNG-KYO SEO ${ }^{4}$ and SOON-CHEOL AHN ${ }^{3}$ \\ ${ }^{1}$ Department of Herbal Formula, Medical Research Center (MRC-GHF), \\ College of Oriental Medicine, Daegu Haany University, Gyeongsan, Republic of Korea; \\ ${ }^{2}$ Korean Medicine (KM)-Application Center, Korea Institute of Oriental Medicine (KIOM), \\ Daegu, Republic of Korea; \\ ${ }^{3}$ Department of Microbiology and Immunology, \\ Pusan National University School of Medicine, Yangsan, Republic of Korea; \\ ${ }^{4}$ School of Life Sciences, Ulsan National Institute of Science and Technology, Ulsan, Republic of Korea; \\ ${ }^{5}$ Department of Infectious Diseases, Armed Forces Medical Research Institute, Daejeon, Republic of Korea
}

\begin{abstract}
Background/Aim: Chemotherapy is a critical option for cancer treatment. However, consistent exposure to chemotherapeutic drugs promotes chemoresistance in cancer cells through diverse mechanisms. Accordingly, we investigated whether salinomycin, a monocarboxylic ionophore, could induce apoptosis in aggressive breast cancer cells or not, as well as its underlying mechanism. Materials and Methods: Using salinomycin on two breast cancer cell lines, MCF-7 cells and MDA-MB-231 cells, cell viability, annexin V/propidium iodide staining, acridine orange staining, caspase-3/9 activity, reactive oxygen species (ROS) and mitochondrial membrane potential (MMP) were assayed. Results: In this study, salinomycin induced apoptosis and autophagy in MDA-MB-231 cells. Salinomycin-mediated ROS production led to mitochondrial dysfunction in MDA-MB-231 cells. Interestingly, treatment of $\mathrm{N}$-acetyl-L-cysteine (NAC), a scavenger of ROS, attenuated salinomycin-induced apoptosis and autophagy. Moreover, autophagy inhibition is involved in acceleration of apoptosis induced by salinomycin. Conclusion: Salinomycin
\end{abstract}

\footnotetext{
*These Authors contributed equally to this study.
}

Correspondence to: Professor Soon-Cheol Ahn, Department of Microbiology and Immunology, Pusan National University School of Medicine, Yangsan 50612, Republic of Korea. Tel +82 515108092, Fax: +82 553828090, e-mail: ahnsc@pusan.ac.kr

Key Words: ROS, autophagy, apoptosis, breast cancer, chemoresistance, salinomycin. induced apoptosis and ROS production, that were blocked by autophagy, thus resulting in protecting cancer cells. This crosstalk of two different physiological responses (autophagy and apoptosis) induced by salinomycin might play pivotal roles in the relationship between autophagy and apoptosis of cancer cells.

In females, breast cancer is the most frequent cancer and a leading cause of death (1). Approximately, 10-15\% of breast cancer cases belong to the triple-negative breast cancer group, showing poor expression of estrogen, progesterone and human epidermal growth factor receptor 2 (HER-2) membrane receptors. Also, this type of cancer is highly aggressive and poorly responds to hormonal therapies. Currently, there is no targeted-therapy to prevent the reoccurrence of this cancer except traditional chemotherapies (2). Therefore, the development of novel therapies and chemotherapeutic drugs are required to improve the chemotherapeutic approach, especially for cases irresponsive to hormone therapies.

In a variety of cellular processes, reactive oxygen species (ROS) act as signaling molecules in apoptotic signaling pathways in addition to by-products of cellular reactions. Recently, it was reported that ROS are central molecules acting in common with apoptosis and autophagy $(3,4)$. ROS regulate cell growth and survival; however, an immoderate production of ROS causes irrevocable cellular damage, inducing apoptosis and/or autophagy. Also, it has been reported that ROS suppress PI3K/AKT/mTOR signaling (5) and activate mitogen-activated protein kinases (MAPK) signaling, including of $\mathrm{C}$-jun $\mathrm{N}$-terminal kinase (JNK), p38 
and extracellular signal-regulated kinases (ERK) (6). Of note, $\mathrm{PI} 3 \mathrm{~K} / \mathrm{AKT} / \mathrm{mTOR}$ inactivation and MAPK activation induced by intracellular ROS have been reported during apoptosis and autophagy. ROS-induced autophagy improves cell survival by promoting degradation of unnecessary components.

Apoptosis is an evolutionary conserved process that is tightly regulated not only to maintain cellular homeostasis but also to suppress tumor development (7). During apoptosis, serial events happen, including cell shrinkage with nuclear and chromatin condensation, membrane blebbing and DNA degradation, as well as generation of apoptotic bodies. Many reports show that cellular stresses, such as ROS, ultraviolet radiation (UV), viral infection and anticancer agents have been known to induce apoptosis $(8,9)$. In addition, autophagy is a self-degradable catabolic mechanism associated with the degradation of unnecessary or dysfunctional components (10). Also, it is considered as a survival mechanism to deal with stresses, such as nutrient deprivation, endoplasmic reticulum (ER) stress, pathogen infection or hypoxia (11). On the other hand, when cells are under harsh stress or deficient in apoptotic processes, autophagy may take part in cell death (12). More recently, several papers reported the roles of autophagy in chemoresistant cancer and the interesting regulation in the process of crosstalk between apoptosis and autophagy (13, 14). During cancer cell nutritional deprivation, cells undergo apoptotic death or autophagy can retard the activation of apoptosis pathway (15). In spite of its importance during cancer therapy to control cancer cell death, molecular pathways are poorly understood and the therapeutic methods controlling autophagy are not yet developed.

Salinomycin is a monocarboxylic ionophore isolated from Streptomyces albus that has been used as antibiotic $(16,17)$. Recently, ionophores are highlighted as a novel class of anticancer agents that can selectively kill cancer cells (18). Several reports showed that salinomycin treatment causes cell cycle arrest and apoptosis via ROS-mediated mitochondrial pathways in a variety of cancer cells $(3,19)$. Salinomycin-mediated apoptosis in these cells is independent of conventional cell death signaling pathway, such as the p53 tumor suppressor protein (20). This drug also triggers apoptosis by overcoming ATP-binding cassette (ABC) transporter-mediated multidrug resistance observed in many cancer cells $(21,22)$. In addition, salinomycin induces activation of autophagy with concomitant activation of AMPactivated protein kinase pathway or activation of ER stress in human cancer cells (23).

This study aims to explore the effective anticancer activity of salinomycin by examining its effects on the induction of cell death and autophagy in chemoresistant MDA-MB 231 cells. Our results demonstrate that salinomycin suppressed the viability of MDA-MB 231 cells by modulating apoptosis and autophagy. The blockage of autophagy pathway enhanced apoptosis in salinomycin-treated cells, which confirmed the ROS by crosstalk between salinomucininduced autophagy and apoptosis. Thus, a therapeutic approach inhibiting autophagy can be a promoting strategy to improve cancer chemoresistance.

\section{Materials and Methods}

Reagents and antibodies. Salinomycin, 2'-7'-dichlorodihydrofluorescein diacetate (DCF-DA), 3-methyladenine (3-MA), 3,3dihexyloxacarbocyanine $\left(\mathrm{DiOC}_{6}\right), 3-(4,5$-Dimethyl-thiazol-2-yl)-2,5diphenyl-etrazolium bromide (MTT), 4', 6-diamidino-2-phenylindole dihydrochloride (DAPI), acridine orange (AO), Fluo-3/AM, $\mathrm{N}$ acetyl-L-cystein (NAC) and verapamil were purchased from Sigma Chemical Co. (St. Louis, MO, USA). FITC Annexin V Apoptosis Detection Kit was obtained from BD Biosciences (San Jose, CA, USA). Antibodies against LC-3, Beclin-1, $\beta$-actin, Bax, Bcl-2, caspase-9, caspase-3, Poly (ADP-ribose) polymerase (PARP) were purchased from Cell Signaling Technology (Beverly, MA, USA) and Santa Cruz Biotechnology (Santa Cruz, CA, USA), respectively. The anti-mouse $\operatorname{IgG}$ and anti-rabbit secondary antibodies raised in goat were purchased from Enzo Life Science (Farmingdale, NY, USA). The FITC-conjugated anti-rabbit IgG antibody was purchased from BioFX Laboratories (Owings Mills, MD, USA).

Cell lines and cell culture. Human breast cancer cell lines, MCF-7 and MDA-MB-231, were obtained from the American Type Culture Collection (Manassas, VA, USA). Cells were maintained in DMEM (WelGENE Inc., Daejeon, Korea) supplemented with 10\% fetal bovine serum (FBS) (WelGENE Inc.), 100 units $/ \mathrm{ml}$ of penicillin and $100 \mu \mathrm{g} / \mathrm{ml}$ of streptomycin (WelGENE Inc.) at $37^{\circ} \mathrm{C}$ in a humidified atmosphere with $5 \% \mathrm{CO}_{2}$.

Cell viability analysis. Human breast cancer MCF-7 and MDA-MB231 cells were seeded in 48-well plates at a density of $1 \times 10^{4} /$ well. Cells were treated with various concentrations of salinomycin for the indicated time intervals. Next, MTT solution $(0.5 \mathrm{mg} / \mathrm{ml})$ was added to the culture medium for a further $4-\mathrm{h}$ incubation at $37^{\circ} \mathrm{C}$ and $5 \%$ $\mathrm{CO}_{2}$ atmosphere. Then cells were dissolved in dimethyl sulfoxide (DMSO) and assays were read at $570 \mathrm{~nm}$ using an ELISA reader (VERSA $_{\max }$ microplate reader; Molecular Devices, Toronto, ONT, Canada). Cell viability is shown as relative percentage to control.

Annexin V/propidium iodide (PI) assay. For the detection of apoptotic cells, FITC Annexin V Apoptosis Detection Kit was used (BD Biosciences). Briefly, breast cancer MCF-7 and MDA-MB-231 cells were cultured in 6-well plates at a density of $5 \times 10^{4} /$ well. After treatment with salinomycin, cells were harvested and then mixed in $100 \mu \mathrm{l}$ of $1 \mathrm{X}$ binding buffer and stained with an Annexin-V/PI at room temperature for $15 \mathrm{~min}$. The stained cells were analyzed by flow cytometry (FACSCalibur; Becton Dickinson, Franklin Lakes, NJ, USA) and the amount of apoptotic cells were calculated using Cell Quest Pro software on Mac ${ }^{\circledR}$ OS 9 (Becton Dickinson).

Measurement of mitochondrial membrane potential. Mitochondrial membrane potentials (MMPs, $\Delta \Psi_{m}$ ) were determined by retention of the $\mathrm{DiOC}_{6}$ dye (3). Briefly, MDA-MB-231 cells were cultured in 6 -well plates at a density of $5 \times 10^{4} /$ well. After treatment with salinomycin, cells were incubated with $100 \mathrm{nM} \mathrm{DiOC}_{6}$ (3) at $37^{\circ} \mathrm{C}$ for $30 \mathrm{~min}$. Cells were washed twice with PBS and analyzed with 
flow cytometry and then calculated using Cell Quest software (Becton Dickinson).

Measurement of intracellular ROS generation. Intracellular ROS generation was measured using the DCF-DA fluorescent dye. MDAMB-231 cells were cultured in 6-well plates at a density of $5 \times 10^{4} /$ well. After treatment with salinomycin, the cells were incubated with $10 \mu \mathrm{M}$ DCF-DA at $37^{\circ} \mathrm{C}$ for $30 \mathrm{~min}$. Cells were washed twice with PBS and analyzed with flow cytometry and then calculated using Cell Quest software (Becton Dickinson).

Analysis of intracellular $\mathrm{Ca}^{2+}$ concentration. Intracellular $\mathrm{Ca}^{2+}$ levels were determined using the $\mathrm{Ca}^{2+}$-sensitive fluorescence dye Fluo-3/AM. MDA-MB-231 cells were cultured in 6-well plates at a density of $5 \times 10^{4} /$ well. After exposure to salinomycin at various concentrations for $48 \mathrm{~h}$, cells were centrifuged, washed twice with PBS and then incubated with $5 \mu \mathrm{M}$ Fluo-3/AM at $37^{\circ} \mathrm{C}$ for $30 \mathrm{~min}$. Washed cells were subjected to flow cytometry and then calculated using Cell Quest software (Becton Dickinson).

Detection of acidic vesicular organelles. To determine the acidic vesicular organelles, MDA-MB-231 cells were cultured in a glassbottom dish. After treatment with salinomycin for indicated interval times, cells were stained with $1 \mu \mathrm{M}$ acridine orange (AO) at $37^{\circ} \mathrm{C}$ in the dark for $20 \mathrm{~min}$. Then, cells were washed with PBS and visualized using a laser scanning confocal microscope (Olympus Fluoview FV1000; Olympus, Tokyo, Japan). Also, to quantify the number of acidic vesicles, cells were harvested and washed with PBS. Then, cells were analyzed by flow cytometry and calculated using Cell Quest software (Becton Dickinson).

Immunofluorescence for $L C$ - 3 . The levels of LC-3 expression were determined using immunofluorescence analysis. MDA-MB-231 cells were seeded on a glass-bottom dish. After treatment with salinomycin with or without $1 \mathrm{mM} 3$-MA for $48 \mathrm{~h}$, cells were fixed with $3 \%(\mathrm{v} / \mathrm{v})$ paraformaldehyde for $10 \mathrm{~min}$. After fixation, the cells were permeabilized with $0.5 \%(\mathrm{v} / \mathrm{v})$ Triton X-100 for $10 \mathrm{~min}$ and blocked with $3 \%(\mathrm{w} / \mathrm{v})$ bovine serum albumin (BSA) for $2 \mathrm{~h}$ at room temperature. After blocking, cells were incubated with primary LC-3 antibody (1:400 diluted in BSA buffer) at room temperature for $2 \mathrm{~h}$ and then reacted with FITC-conjugated antirabbit IgG secondary antibody. DAPI was used to stain the nuclei. Samples were visualized with a laser scanning confocal microscope.

Western blot analysis. Cell extracts were prepared by incubating the cells in lysis buffer ( $150 \mathrm{mM} \mathrm{NaCl}, 10 \mathrm{mM}$ Tris (pH 7.4), $5 \mathrm{mM}$ EDTA (pH 8.0), $1 \%$ Triton X-100, 1 mM PMSF, $20 \mu \mathrm{g} / \mathrm{ml}$ aprotinin, $50 \mu \mathrm{g} / \mathrm{ml}$ leupeptin, $1 \mathrm{mM}$ benzidine, $1 \mathrm{mg} / \mathrm{ml}$ pepstatin, $8 \mathrm{mM}$ sodium pyrophosphate and $20 \mathrm{mM} \beta$-glycerophosphate). Forty micrograms of protein was electrophoretically separated using sodium dodecyl sulfate-polyacrylamide gel electrophoresis (SDSPAGE) on $8-15 \%$ gel and transferred to a polyvinylidenefluoride (PVDF) membrane. After blocking with TBS-T buffer $(20 \mathrm{mM}$ Tris (pH 7.4), $150 \mathrm{mM} \mathrm{NaCl}, 0.1 \%$ Tween 20) containing 5\% skim milk, membranes were incubated with primary and secondary antibodies. The membranes were then washed with TBS-T buffer and visualized with ECL Western blot analysis detection reagents. The density of each band was determined with a fluorescence scanner (LAS 3000; Fuji Film, Tokyo, Japan) and analyzed with Multi Gauge V3.0 software (Fuji Film).
Statistical analysis. Experiments were repeated at least three times with consistent results. Unless otherwise stated, data are expressed as the mean \pm standard deviation (SD). Analysis of variance (ANOVA) was used to compare experimental groups to control values. Comparisons between multiple groups were performed using Tukey's multiple comparison tests. Results were statistically significant at $p<0.05$.

\section{Results}

Salinomycin inhibited cell growth and induced apoptosis in human breast cancer cells. Since salinomycin was reported to mediate apoptosis in many human cancer cells $(3,19,20)$, we investigated the mechanism autophagy follows for protection against apoptosis in salinomycin-treated chemoresistant breast cancer cells. In order to determine the growth inhibition by salinomycin, breast cancer cells MDA-MB-231 and MCF-7 were treated with salinomycin for 24 and $48 \mathrm{~h}$, respectively, and viabilities were measured by MTT assay. MDA-MB-231 cells were shown relatively less sensitive to salinomycin than MCF-7 cells (Figure 1A and B).

Next, we investigated whether the observed inhibition of cell viability upon salinomycin treatment in both MDA-MB231 and MCF-7 cells was associated with induction of apoptosis. Flow cytometry analysis of Annexin V and PI staining was used to determine the percentage of apoptotic cells induced by various salinomycin concentrations. By this approach, $36.76 \%$ and $25.88 \%$ of apoptotic cells were detected in $2.5 \mu \mathrm{M}$ salinomycin-treated MCF-7 and MDA-MB-231 cells, respectively (Figure $1 C$ ). These results indicated that apoptotic resistance of MDA-MB-231 cells is higher than that of MCF-7 cells. To further understand the molecular basis of salinomycin-induced apoptosis, changes of the expression of apoptosis-related proteins, such as Bax, Bcl-2, pro-caspase-3 and PARP, were evaluated by western blot analysis. As shown in Figure 1D, the pro-apoptotic protein Bax was induced, whereas the expression of anti-apoptotic protein Bcl-2 was inhibited in dose-dependent manner in MDA-MB231 cells. This suggested that the increase of $\mathrm{Bax} / \mathrm{Bcl}-2$ ratios might be involved in apoptosis induced by salinomycin. Furthermore, the pro-form of caspase-3, one of key regulators in apoptosis, was reduced in a dose-dependent manner in MDA-MB-231 cells, while higher cleavage of PARP was detected in elevated concentrations of salinomycin (Figure 1D).

Next, we quantified salinomycin-mediated mitochondrial dysfunction at indicated concentrations. As shown in Figure $1 \mathrm{E}$, the mitochondrial membrane potential was increased in dose-dependent manner in MDA-MB-231 cells. These results show that salinomycin can initiate a cascade of events that provoke the mitochondrial-dependent apoptotic pathway in MDA-MB-231 cells.

ROS production is associated with salinomycin-induced apoptosis of MDA-MB-231 cells. Several studies reported that 

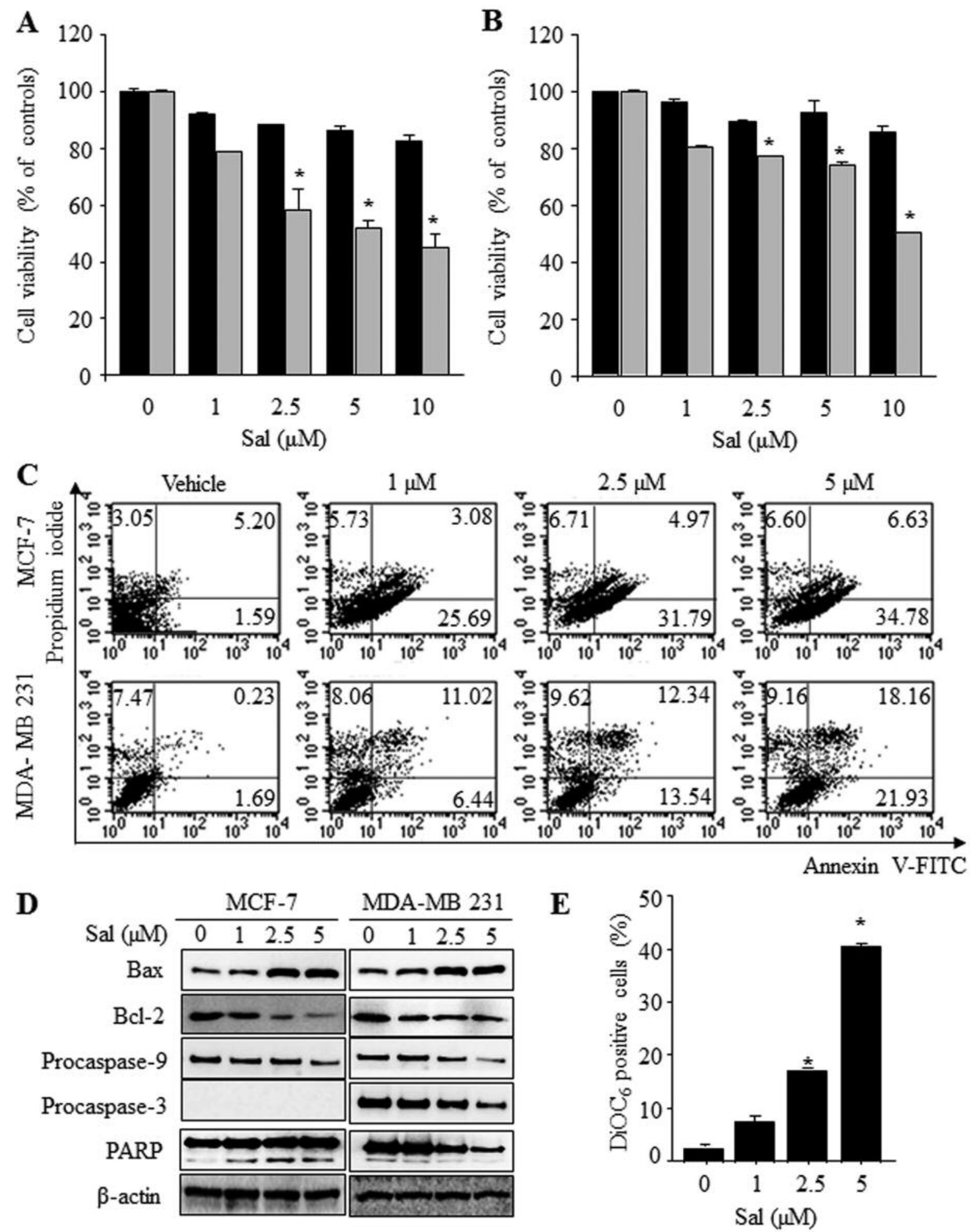

Figure 1. Salinomycin inhibits cell growth and induces apoptosis in breast cancer cells. (A) Cell growth inhibition: MCF-7 and MDA-MB-231 cells were treated with salinomycin at different time points. Cell viability was determined by 3-(4,5-dimethyl-thiazol-2-yl)-2,5-diphenyl-etrazolium bromide (MTT) assay. (B) Apoptosis induction: MCF-7 and MDA-MB-231 cells were treated with various concentrations of salinomycin for 48 h. (C) Bcl-2 family and Pro-caspase-3 and poly (ADP-ribose) polymerase (PARP) expression in total cell lysates were subjected to SDS-PAGE for western blot analysis. (D) Mitochondrial membrane potential (MMP): MDA-MB-231 cells were treated with salinomycin for 48 h. MMP changes were determined by DiOC 6 fluorescence, measured by flow cytometry analysis. All data are representative of at least three independent experiments. Data show mean \pm SD. *p<0.05. 
ROS function as key regulator in chemotherapeutic druginduced apoptosis in a variety of cell types. To elucidate whether ROS production is associated with salinomycininduced apoptosis of MDA-MB-231 cells, the level of ROS was measured at various time points of salinomycin treatment by examining the fluorescence intensity of DCH-DA-stained cells. A representative flow cytometry fluorescence pattern showed that intracellular ROS level increased after $24 \mathrm{~h}$ of salinomycin treatment, with its maximal level being detected at $48 \mathrm{~h}$ (Figure 2A). As expected, cellular pretreatment with $\mathrm{N}$-acetyl cysteine (NAC), a known scavenger of ROS, attenuated the level of ROS induced by salinomycin (Figure $2 \mathrm{~A}$, right panel). Furthermore, the addition of NAC significantly reduced the apoptotic cells from $35.50 \%$ to $23.86 \%$ (Figure 2B). Also, pretreatment with NAC restored salinomycin-induced caspase- 3 activation and PARP cleavage (Figure 3A). These findings suggest that intracellular ROS production is closely linked to caspase-3 activation-dependent apoptosis of MDA-MB-231 cells.

Intracellular calcium is one of the physiological regulators associated with ROS production and apoptosis. Fluo-3/AM, a $\mathrm{Ca}^{2+}$-sensitive fluorescence probe was used to monitor changes in the intracellular $\mathrm{Ca}^{2+}$ level. The fluorescence emitted from salinomycin-treated cells was increased in a dose-dependent manner (Figure 2C), indicating a salinomycin-induced elevation of intracellular $\mathrm{Ca}^{2+}$ flux. In contrast, the L-type calcium channel blocker, verapamil, significantly reduced the elevated $\mathrm{Ca}^{2+}$ flux (Figure 2C). Moreover, this blocker also reduced apoptotic cells from $18.71 \%$ to $9.33 \%$ (Figure 2D), as well as salinomycininduced caspase- 3 activation and PARP cleavage (Figure 3B). These findings suggested that the regulation of intracellular calcium is closely linked to salinomycin-induced apoptosis.

To examine the correlation between ROS production and intracellular $\mathrm{Ca}^{2+}$ concentration, we measured levels of ROS and $\mathrm{Ca}^{2+}$ in the presence or absence of NAC and verapamil. NAC pretreatment prevented increase of $\mathrm{Ca}^{2+}$ concentration, whereas ROS production did not change by verapamil (Figure 3C and 3D) indicating that ROS play a pivotal role as upstream regulators in $\mathrm{Ca}^{2+}$ modulation in salinomycintreated conditions. In addition, to determine whether $\mathrm{Ca}^{2+}$ concentration and ROS production influence mitochondrial dysfunction, MMP were assessed after pretreatment with NAC or verapamil. As shown in Figure 3E and 3F, NAC did not influence MMP, whereas verapamil blocked salinomycinmediated increase of MMP (Figure 3F). Taken together, the accumulation of ROS preceded the elevation of intracellular $\mathrm{Ca}^{2+}$, followed by the mitochondrial dysfunction.

Salinomycin induced autophagy in breast cancer MDA-MB231 cells. Next, we hypothesized that autophagy could be induced in salinomycin-treated breast cancer cells to survive from apoptotic death. For verification, autophagy was analyzed by accumulation of acidic vesicular organelles (AVOs) stained with AO. As shown in Figure 4A, salinomycin caused more accumulation of AVOs in MDAMB-231 cells, compared to that in MCF-7 cells. Additionally, the formation of AVOs was confirmed using confocal microscopy in MDA-MB-231 cells. In contrast to control cells showing green fluorescence with minimal red fluorescence, salinomycin-treated cells showed an increased level of red fluorescence in a time-dependent manner (Figure 4B). In addition, autophagy-specific markers, LC-3 and Beclin-1, were also used to examine the autophagic level by western blot analysis. The level of LC-3-I/II converse forms was increased in MDA-MB-231 cells in a time-dependent manner, while Beclin-1 levels were not changed (Figure 4C). These results indicated that salinomycin induces autophagosomes in MDA-MB-231 cells through a Beclin-1independent pathway.

Subsequently, an autophagy inhibitor, 3-methyladenine (3MA), was used to further confirm the salinomycin-induced autophagy. Indeed, the autophagic cells possessing AVOs were decreased by 3-MA treatment in salinomycin-treated cells, thus indicating that salinomycin could induce autophagy in MDA-MB-231 cells (Figure 5A). Additionally, LC-3 immunofluorescence and Western blot analysis were also performed. The punctum of GFP-LC-3 was enhanced by salinomycin treatment, whereas the punctum was blocked in 3-MA pretreated cells (Figure 5B).

As a next step, we examined the effects of autophagy inhibition on salinomycin-induced apoptosis in MDA-MB231 cells using annexin V/PI staining. Under apoptotic conditions induced by salinomycin, 3-MA strengthened salinomycin-mediated apoptosis (Figure 5C). Moreover, caspase- 3 activation and PARP cleavage were also increased, whereas the conversion of LC-3-I/II was reduced (Figure 5D). Taken together, salinomycin-induced autophagy has a protective role against apoptosis. On the other end, inhibition of autophagy enhances apoptosis via caspase-3-dependent pathway in MDA-MB-231 cells.

Autophagy inhibition stimulated ROS production in breast cancer MDA-MB-231 cells. As mentioned above, salinomycin induces apoptosis via accumulated ROS and mitochondrial dysfunction in MDA-MB-231 cells. Also, recent studies reported that ROS might play roles as signaling mediators in apoptosis and autophagy. Therefore, we investigated whether ROS production can induce AVOs accumulation in salinomycin-treated MDA-MB-231 cells. By comparing the autophagic cell rate, addition of NAC significantly reduced AVOs-positive cells from $33.68 \%$ to $12.35 \%$ (Figure 6A). Furthermore, salinomycin-induced LC3 conversion was inhibited by NAC (Figure 6B). These results indicate that ROS production mediates the salinomycin-induced autophagy. Next, we evaluated the 
$\mathbf{A}$

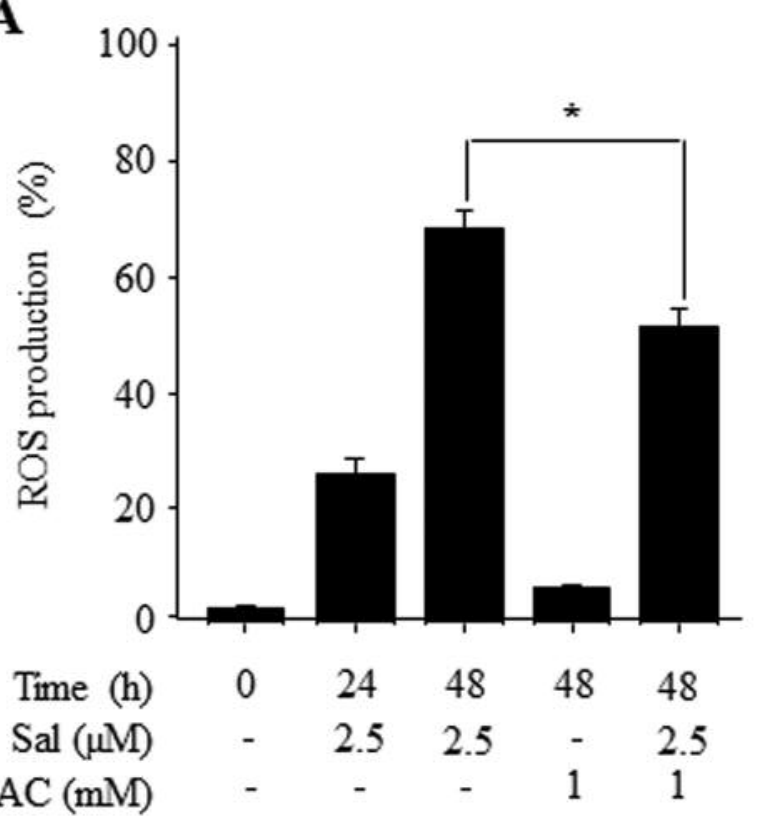

B

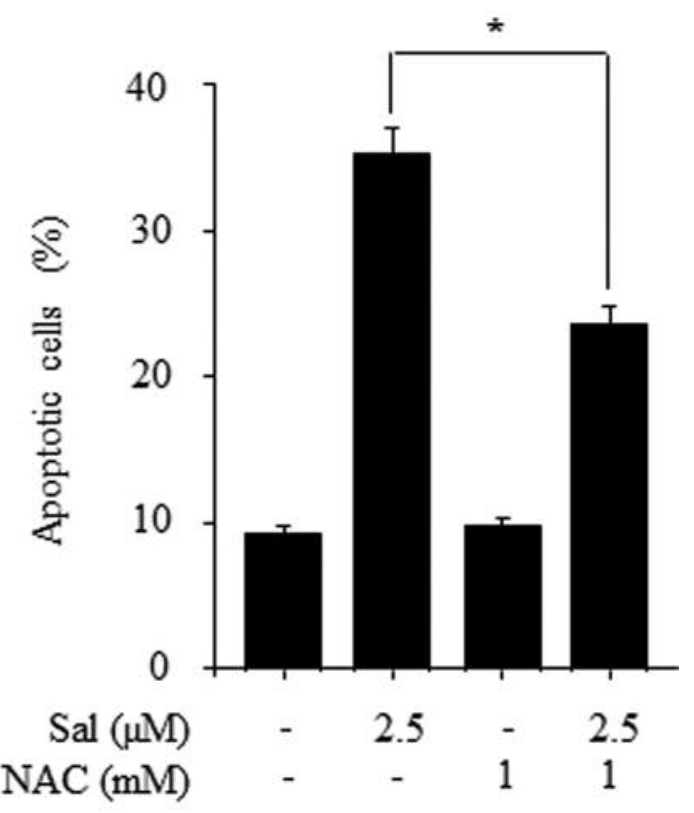

D

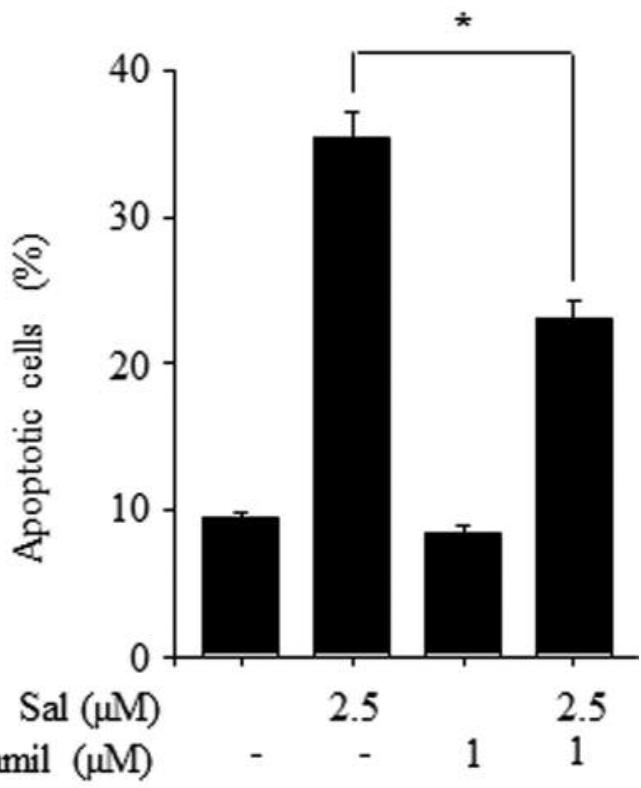

Figure 2. Salinomycin induces ROS production upstream of $\mathrm{Ca}^{2+}$ homeostasis via mitochondrial dysfunction in breast cancer MDA-MB-231 cells. (A) Intracellular ROS levels: MDA-MB-231 cells were treated with salinomycin for indicated time periods with or without prior $1 \mathrm{~h}$ incubation with $1 \mathrm{~m}$ $M N A C$. The DCF-DA fluorescence intensity was detected by flow cytometry. (B) Effects of NAC in salinomycin-induced apoptosis: MDA-MB-231 cells

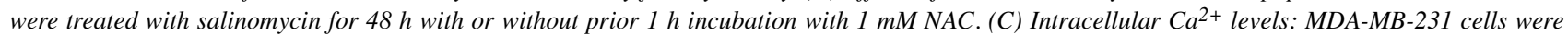
treated with salinomycin for $48 \mathrm{~h}$ with or without prior $1 \mathrm{~h}$ incubation with $1 \mu \mathrm{M}$ verapamil. The Fluo-3/AM fluorescence intensity was detected by flow cytometry. (D) Effects of verapamil in salinomycin-induced apoptosis: MDA-MB-231 cells were treated with salinomycin for $48 \mathrm{~h}$ with or without prior $1 \mathrm{~h}$ incubation with $1 \mu \mathrm{M}$ verapamil. All data are representative of at least three independent experiments. Data show mean $\pm S D$. ${ }^{*} p<0.05$.

reciprocal effect between autophagy and ROS production in salinomycin-treated cells. When autophagy was inhibited by 3-AM treatment, more ROS was produced in salinomycin- treated cells (Figure 6C). Moreover, additional production of ROS by 3-MA treatment was also efficiently recovered by NAC pre-treatment. Next, we examined whether salinomycin- 
$\mathbf{A}$

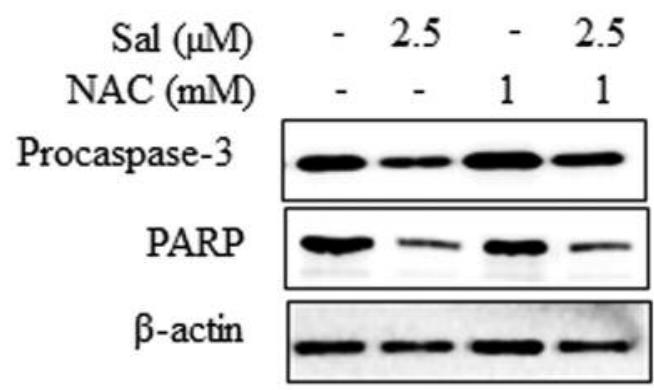

C

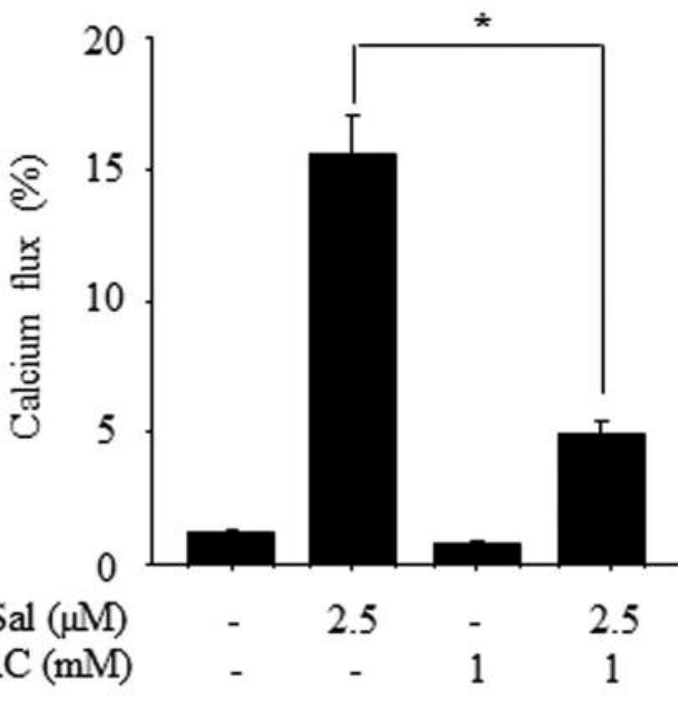

$\mathbf{E}$

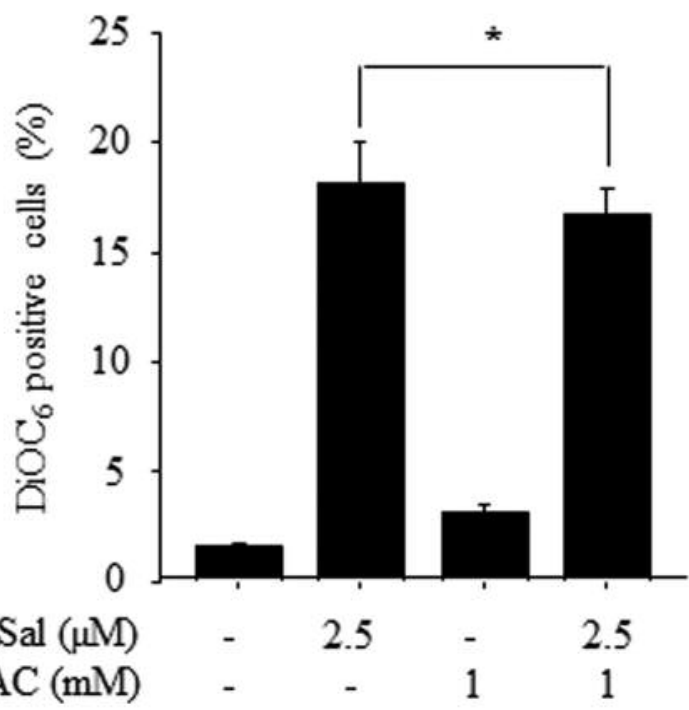

B

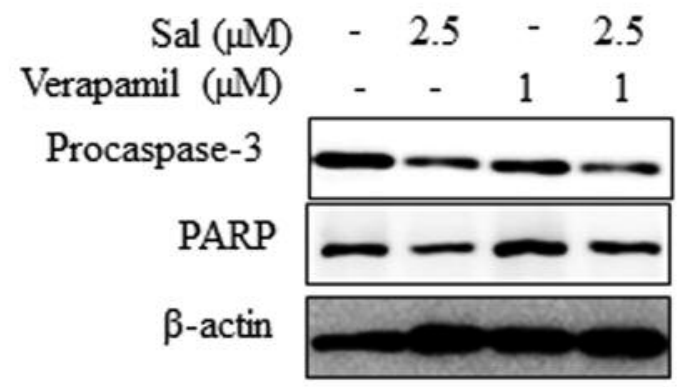

D

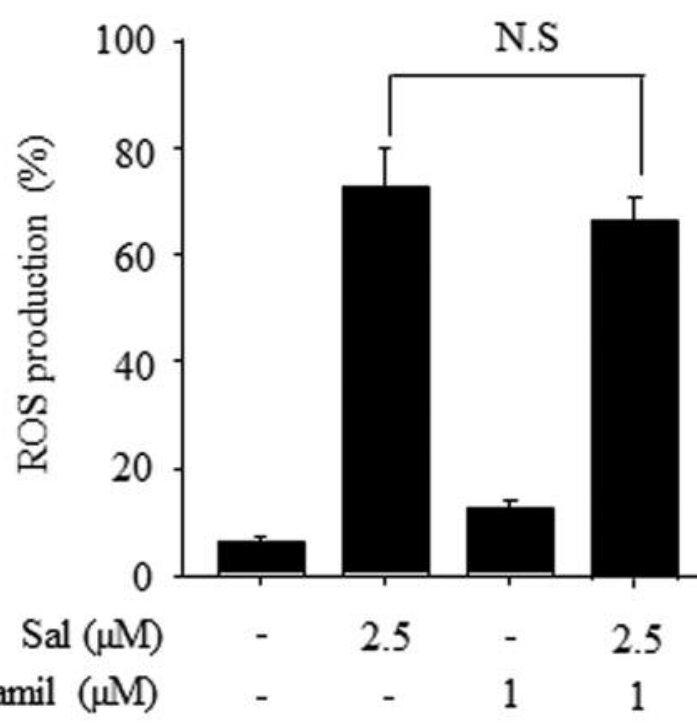

$\mathbf{F}$

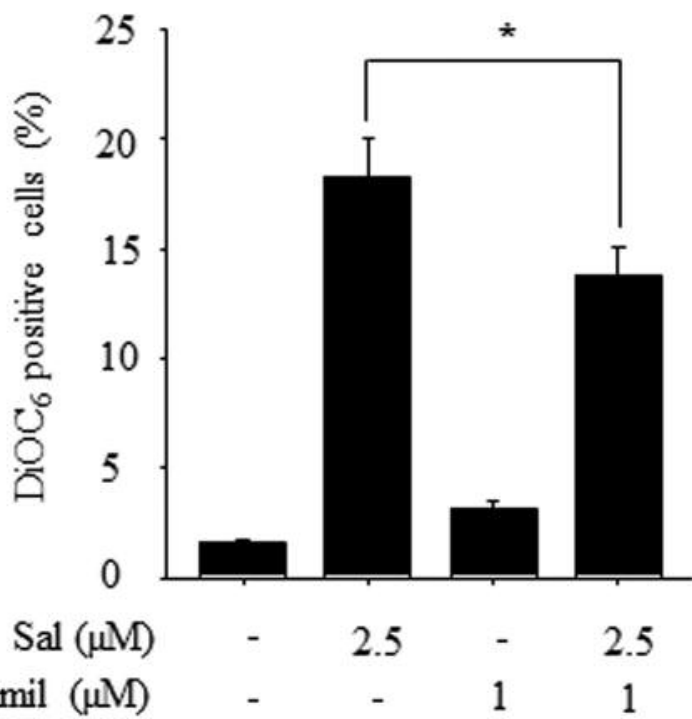

Figure 3. Salinomycin-induced apoptosis involves ROS production upstream of $\mathrm{Ca}^{2+}$ homeostasis via mitochondrial dysfunction in MDA-MB-231 cells. (A) Effects of pro-caspase-3 and poly (ADP-ribose) polymerase (PARP) expression. (B) Effects of verapamil in pro-caspase-3 and poly (ADPribose) polymerase (PARP) expression. (C) Effects of NAC in intracellular Ca ${ }^{2+}$ levels. (D) Effects of NAC in MMP. (E) Effects of verapamil in intracellular ROS levels. $(F)$ Effects of verapamil in MMP: MDA-MB-231 cells were treated with salinomycin for $48 \mathrm{~h}$ with or without prior $1 \mathrm{~h}$ incubation with $1 \mathrm{mM} N A C$ or $1 \mu \mathrm{M}$ verapamil. The DCF-DA and Fluo-3/AM and DiOC 6 fluorescence intensity was detected by flow cytometry. All data are representative of at least three independent experiments. Data show mean $\pm S D$. ${ }^{*} p<0.05 ;$ N.S., not significant. 

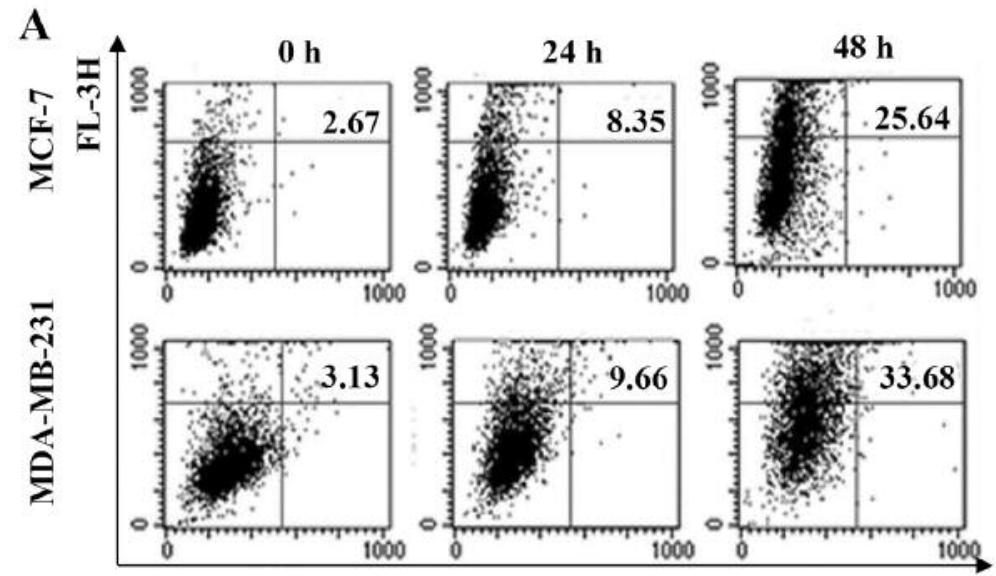

FL-1H

B

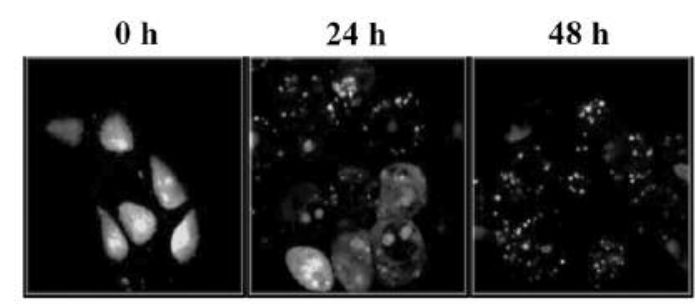

C

Salinomycin in MDA MB-231

Figure 4. Salinomycin induces autophagy in breast cancer cells. (A) Acidic vacuoles' detection: After treatment with salinomycin for indicated times, cells were stained with $1 \mu \mathrm{M}$ acridine orange $(A O)$ at $37^{\circ} \mathrm{C}$ in the dark for $20 \mathrm{~min}$. Then, cells were washed with PBS and, subsequently, analyzed by flow cytometry and visualized using with a laser scanning confocal microscope. (B) AO staining. (C) LC-3 and Beclin-1 expression: After treatment with salinomycin, total cell lysates were subjected to SDS-PAGE for western blot analysis.

induced autophagy inhibits apoptosis through regulation of ROS production. As shown Figure 6D, enhancement of salinomycin-induced apoptosis by 3-MA was completely inhibited by ROS prevention. Taken together, these results showed that not only ROS production plays a key role in both autophagy and apoptosis but autophagy inhibition also enhances salinomycin-induced apoptosis by increasing ROS production in MDA-MB-231 cells.

\section{Discussion}

We have previously shown that salinomycin reduces cell viability in a variety of cancer cells $(3,24)$. Salinomycin markedly inhibited the cell growth of diverse cancer cells, including drug-sensitive and drug-resistant cancer cells $(3,19$, $25)$. Since there are no reports on the molecular mechanism(s) on the anticancer effects of salinomycin, we designed studies to evaluate the anticancer properties of salinomycin on the human breast cancer cells MCF-7 and MDA-MB-231. Here, we demonstrate that salinomycin induced ROS-mediated apoptosis in MDA-MB-231 cells, accompanied by increase of autophagy for cancer cell survival.
Consistent with previous reports $(26,27)$, salinomycin inhibited the proliferation of both MCF-7 and MDA-MB-231 breast cancer cells through induction of apoptosis, which was induced by ROS production via mitochondrial dysfunction. Interestingly, cancer cells showed different degrees of sensitivity to salinomycin depending on their hormoneindependent status and chemo-resistance. The apoptotic rates of MDA-MB-231 cells, that are highly hormone-independent and chemo-resistant, were markedly lower than that of hormone-dependent and chemo-sensitive MCF-7 cells. To understand antitumor mechanisms, the relative expression levels of Bcl-2, Bax, caspase-9, caspase-3 and PARP proteins affected by salinomycin were measured by western blot analysis. Our study showed that salinomycin not only downregulated the expression of Bcl-2 and simultaneously enhanced the expression of Bax, which can activate the mitochondrial apoptosis pathway, but also increased the PARP activity in both breast cell lines, which inclined to induce apoptosis.

Our results also suggested that there exists another cytoprotective mechanism involved in the resistance against apoptosis. Salinomycin induced autophagy as evidenced via the occurrence of autophagosomes and acidic vacuoles stained 
A

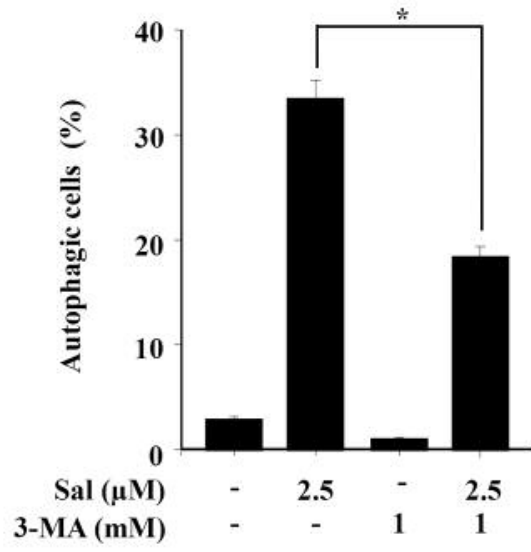

C

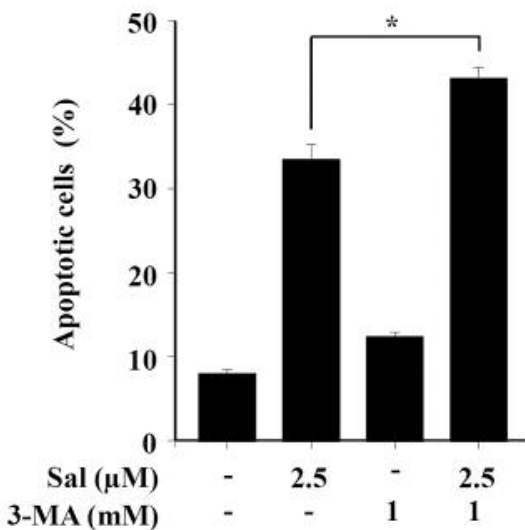

B

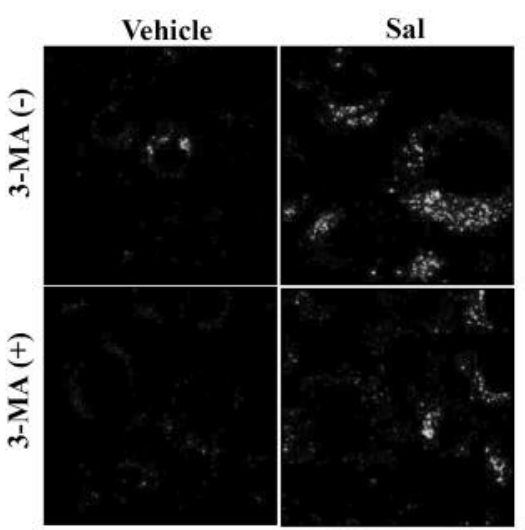

D

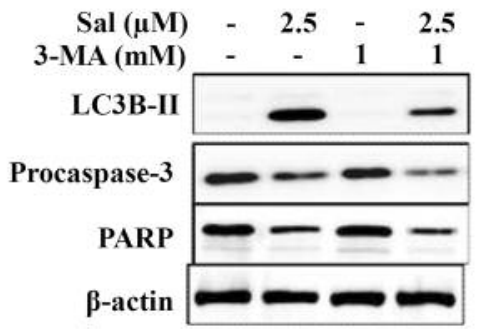

Figure 5. Autophagy inhibition enhances salinomycin-induced apoptosis in breast cancer cells. (A) Effects of 3-MA in salinomycin-induced autophagy: Cells were treated with $2.5 \mu \mathrm{M}$ salinomycin for $48 \mathrm{~h}$, with or without prior $1 \mathrm{~h}$ incubation with $1 \mathrm{mM} 3-\mathrm{MA}$, and then incubated with $1 \mu \mathrm{g} / \mathrm{ml}$ acridine orange in serum-free medium for $20 \mathrm{~min}$. Acidic vesicular organelles (AVOs) were detected by flow cytometry. (B) Immunofluorescence staining for LC-3: Cells were treated with $2.5 \mu \mathrm{M}$ salinomycin for $48 \mathrm{~h}$, with or without prior $1 \mathrm{~h}$ incubation with $1 \mathrm{mM}$ 3-MA. Fluorescence was detected by confocal microscopy. (C) Effects of 3MA in salinomycin-induced apoptosis: Cells were treated with $2.5 \mu M$ salinomycin for $48 \mathrm{~h}$, with or without prior $1 \mathrm{~h}$ incubation with $1 \mathrm{mM}$ 3-MA. Apoptotic cells were detected by flow cytometry. (D) Effects of 3-MA in LC-3, pro-caspase-3 and PARP protein expression: Cells were treated with $2.5 \mu M$ salinomycin for 48 h, with or without prior $1 \mathrm{~h}$ incubation with $1 \mathrm{mM} 3-\mathrm{MA}$, then the proteins were detected by western blot analysis. All data are representative of at least three independent experiments.

with $\mathrm{AO}$ as well as the conversion of LC-3-I to LC-3-II (Figure 4). However, the rates of salinomycin-induced autophagy in MCF-7 cells were lower than those in MDA-MB 231 cells. Also, as expected, salinomycin-induced autophagy and LC-3 conversion were inhibited by autophagy inhibitor 3MA. We also showed that autophagy inhibition enhanced salinomycin-induced apoptosis through caspase activation in MDA-MB-231 cells. Previous reports showed that the autophagy pathway seems to play some role in controlling the susceptibility of the cancer stem cells/progenitor phenotype and actually potentiate salinomycin's toxicity in hypoxic, lowglucose, low-nutrient and growth factor conditions $(18,21)$. These results imply that autophagy might protect cells from cytotoxic effects through blocking apoptosis or killing cells.
Many reports show that autophagy and apoptosis regulate cell death by controlling ROS homeostasis (27, 29). Also, evidence provides the link between apoptosis and autophagy (30). Consistent with previous observations, we also showed that when ROS scavenger NAC was used before salinomycin treatment, autophagic markers, including LC-3 expression, were significantly restored by NAC in MDA-MB-231 cells. In addition, inhibition of autophagy led to an increase in ROS formation and salinomycin-induced apoptosis in MDA-MB-231 cells. These results suggest that ROS production is a primary mechanism in the salinomycin-induced apoptosis and autophagy. Furthermore, inhibition of ROS production decreased caspase- 3 activity and PARP cleavage in these 


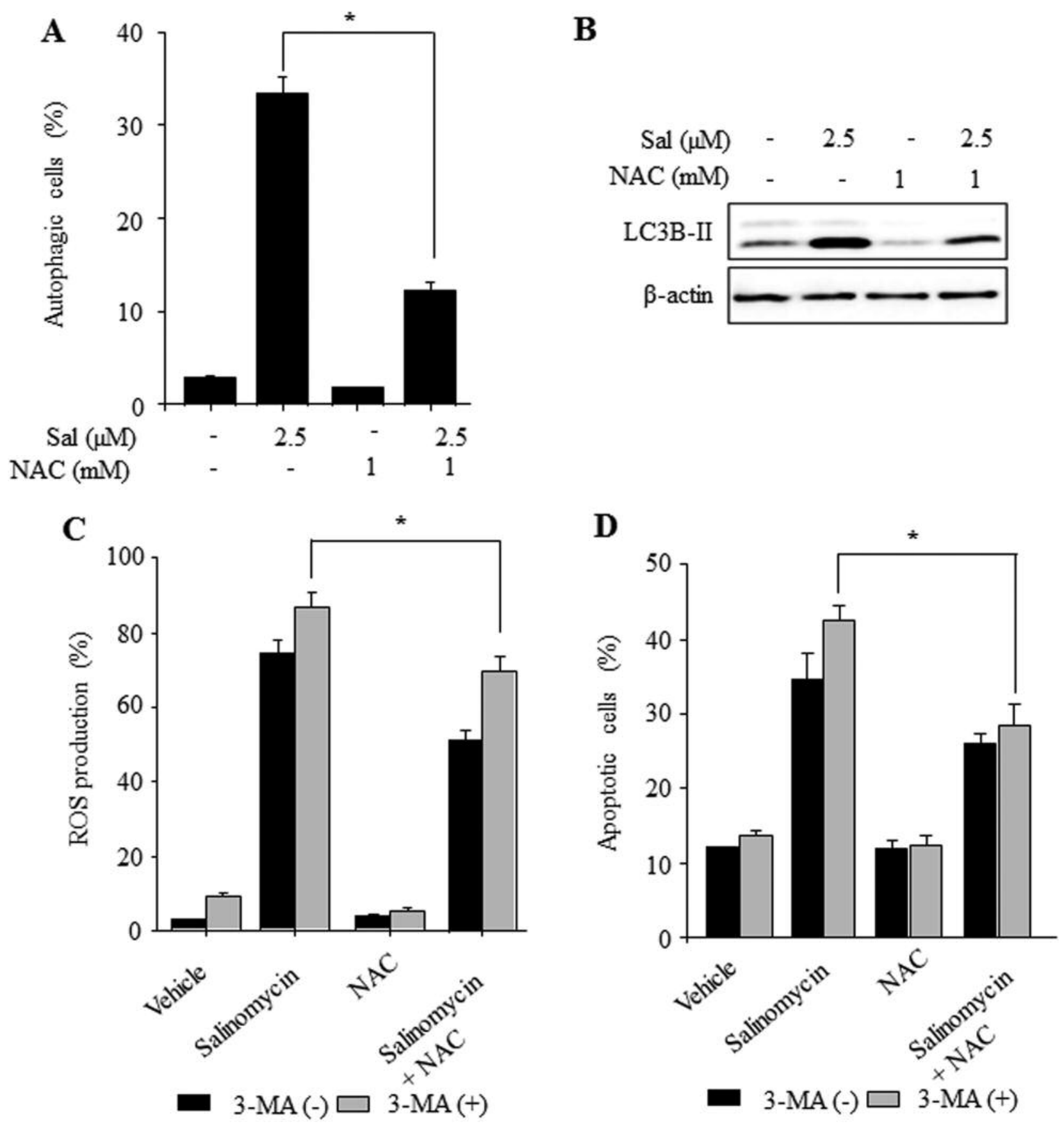

Figure 6. ROS production regulates salinomycin-induced autophagy and apoptosis in breast cancer cells. (A) Effects of NAC in salinomycin-induced autophagy. (B) Effects of NAC in LC-3 protein expression: Cells were treated with $2.5 \mu M$ salinomycin for 48 , with or without prior $1 \mathrm{~h}$ incubation with $1 \mathrm{mM} 3-\mathrm{MA}$, and then incubated with $1 \mu \mathrm{g} / \mathrm{ml}$ acridine orange in serum-free medium for $20 \mathrm{~min}$. acidic vesicular organelles (AVOs). Proteins were detected by flow cytometry and western blot analysis, respectively. (C) Effects of 3-MA in ROS production. (D) Effects of 3-MA in ROSmediated salinomycin-induced apoptosis. Cells were treated with $2.5 \mu \mathrm{M}$ salinomycin for $48 \mathrm{~h}$, with or without prior $1 \mathrm{~h}$ incubation alone or in combination with $1 \mathrm{mM}$ 3-MA or $10 \mu \mathrm{M}$ NAC. Apoptotic cells or DCF-DA-positive cells were detected by flow cytometry. All data are representative of at least three independent experiments. Data show mean $\pm S D .{ }^{*} p<0.05$.

cells. Salinomycin markedly inhibited the cell growth of drug-sensitive cells, as well as drug-resistant cells. The sensitivity to salinomycin is differentially displayed depending on the cell type employed. When salinomycin induced apoptotic cell death, ROS production triggered the mitochondrial apoptotic pathway. Furthermore, the adverse 


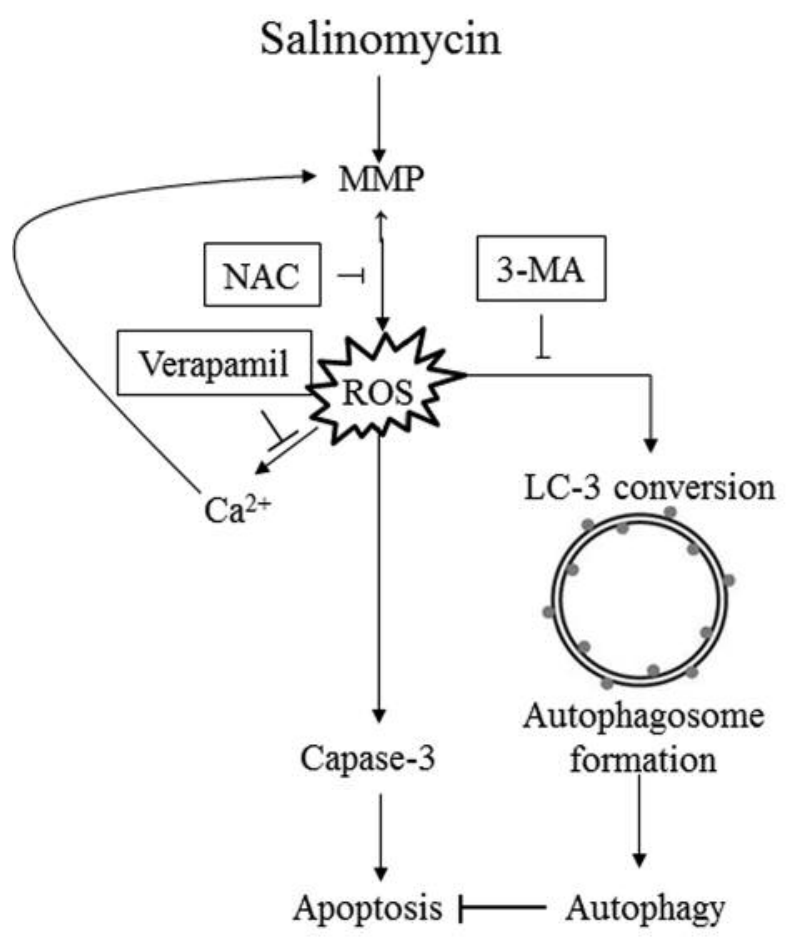

Figure 7. Schematic drawing representing possible mechanisms underlying salinomycin-induced apoptosis and autophagy in breast cancer cells.

effects of autophagy to ROS-mediated apoptosis suggested that autophagy functions as a resistance mechanism against apoptosis (Figure 7). Therefore, we believe that, in cancer chemoprevention or chemotherapy, salinomycin can be a promising compound to control autophagy. Further research to in-depth explore the molecular mechanisms and evaluate salinomycin's anticancer activity in vivo is necessary.

\section{Conflicts of Interests}

The Authors declare that they have no competing interests.

\section{Acknowledgements}

This research was supported by Basic Science Research Program through the National Research Foundation of Korea (NRF) funded by the Ministry of Education (NRF-2013R1A1A2012638) and the Korea Institute of Oriental Medicine funded by the Ministry of Education, Science and Technology (MEST), Republic of Korea (No. K17281).

\section{References}

1 Siegel R, Naishadham D and Jemal A: Cancer statistics, 2013. CA Cancer J Clin 63: 11-30, 2013.

2 Jemal A, Bray F, Center MM, Ferlay J, Ward E and Forman D: Global cancer statistics. CA Cancer J Clin 61: 69-90, 2011.
3 Kim KY, Yu SN, Lee SY, Chun SS, Choi YL, Park YM, Song CS, Chatterjee B and Ahn SC: Salinomycin-induced apoptosis of human prostate cancer cells due to accumulated reactive oxygen species and mitochondrial membrane depolarization. Biochem Biophys Res Commun 413: 80-86, 2011.

4 Kongara S and Karantza V: The interplay between autophagy and ROS in tumorigenesis. Front Oncol 2: 171, 2012.

5 Lee JH, Won YS, Park KH, Lee MK, Tachibana H, Yamada K and Seo KI: Celastrol inhibits growth and induces apoptotic cell death in melanoma cells via the activation ROS-dependent mitochondrial pathway and the suppression of PI3K/AKT signaling. Apoptosis 17: 1275-1286, 2012.

6 Alhosin M, Anselm E, Rashid S, Kim JH, Madeira SV, Bronner $\mathrm{C}$ and Schini-Kerth VB: Redox-sensitive up-regulation of eNOS by purple grape juice in endothelial cells: Role of PI3kinase/Akt, p38 MAPK, JNK, FoxO1 and FoxO3a. PLoS One 8: e57883, 2013.

7 Elmore S: Apoptosis: A review of programmed cell death. Toxicol Pathol 35: 495-516, 2007.

8 Pan MH and Ho CT: Chemopreventive effects of natural dietary compounds on cancer development. Chem Soc Rev 37: 25582574,2008 .

9 Wang ZB, Liu YQ, Zhang Y, Li Y, An XX, Xu H, Guo Y, Jin W, Jiang ZJ and Cui YF: Reactive oxygen species, but not mitochondrial membrane potential, is associated with radiationinduced apoptosis of AHH-1 human lymphoblastoid cells. Cell Biol Int 31: 1353-1358, 2007.

10 Yang $\mathrm{Z}$ and Klionsky DJ: Eaten alive: A history of macroautophagy. Nat Cell Biol 12: 814-822, 2010.

11 Glick D, Barth S and Macleod KF: Autophagy: Cellular and molecular mechanisms. J Pathol 221: 3-12, 2010.

12 Kroemer G and Levine B: Autophagic cell death: The story of a misnomer. Nat Rev Mol Cell Biol 9: 1004-1010, 2008.

13 Suman S, Das TP, Reddy R, Nyakeriga AM, Luevano JE, Konwar D, Pahari P and Damodaran C: The pro-apoptotic role of autophagy in breast cancer. Br J Cancer 111: 309-317, 2014.

14 Young MM, Kester M and Wang HG: Sphingolipids: regulators of crosstalk between apoptosis and autophagy. J Lipid Res 54: 5-19, 2013.

15 Jain MV, Paczulla AM, Klonisch T, Dimgba FN, Rao SB, Roberg K, Schweizer F, Lengerke C, Davoodpour P, Palicharla VR, Maddika S and Los M: Interconnections between apoptotic, autophagic and necrotic pathways: Implications for cancer therapy development. J Cell Mol Med 17: 12-29, 2013.

16 Naujokat C, Fuchs D and Opelz G: Salinomycin in cancer: A new mission for an old agent. Mol Med Rep 3: 555-559, 2010.

17 Pressman BC: Antibiotic models for carrier-mediated transport through membranes. Antimicrob Agents Chemother (Bethesda) 9: 28-34, 1969.

18 Gupta PB, Onder TT, Jiang G, Tao K, Kuperwasser C, Weinberg RA and Lander ES: Identification of selective inhibitors of cancer stem cells by high-throughput screening. Cell 138: 645$659,2009$.

19 Al Dhaheri Y, Attoub S, Arafat K, Abuqamar S, Eid A, Al Faresi $\mathrm{N}$ and Iratni R: Salinomycin induces apoptosis and senescence in breast cancer: Upregulation of $\mathrm{p} 21$, downregulation of survivin and histone $\mathrm{H} 3$ and $\mathrm{H} 4$ hyperacetylation. Biochim Biophys Acta 1830: 3121-3135, 2013. 
20 Kim JH, Chae M, Kim WK, Kim YJ, Kang HS, Kim HS and Yoon S: Salinomycin sensitizes cancer cells to the effects of doxorubicin and etoposide treatment by increasing DNA damage and reducing p21 protein. Br J Pharmacol 162: 773-784, 2011.

21 Kim WK, Kim JH, Yoon K, Kim S, Ro J, Kang HS and Yoon S: Salinomycin, a p-glycoprotein inhibitor, sensitizes radiationtreated cancer cells by increasing DNA damage and inducing G2 arrest. Invest New Drugs 30: 1311-1318, 2012.

22 Riccioni R, Dupuis ML, Bernabei M, Petrucci E, Pasquini L, Mariani G, Cianfriglia M and Testa U: The cancer stem cell selective inhibitor salinomycin is a p-glycoprotein inhibitor. Blood Cells Mol Dis 45: 86-92, 2010.

23 Jangamreddy JR, Ghavami S, Grabarek J, Kratz G, Wiechec E, Fredriksson BA, Rao Pariti RK, Cieslar-Pobuda A, Panigrahi S and Los MJ: Salinomycin induces activation of autophagy, mitophagy and affects mitochondrial polarity: differences between primary and cancer cells. Biochim Biophys Acta 1833: 2057-2069, 2013.

24 Boehmerle W and Endres M: Salinomycin induces calpain and cytochrome c-mediated neuronal cell death. Cell Death Dis 2: e168, 2011.

25 Kim KY, Kim SH, Yu SN, Park SK, Choi HD, Yu HS, Ji JH, Seo YK and Ahn SC: Salinomycin enhances doxorubicin-induced cytotoxicity in multidrug resistant MCF-7/MDR human breast cancer cells via decreased efflux of doxorubicin. Mol Med Rep 12: 1898-1904, 2015.

26 Kim KY, Cho HJ, Yu SN, Kim SH, Yu HS, Park YM, Mirkheshti N, Kim SY, Song CS, Chatterjee B and Ahn SC: Interplay of reactive oxygen species, intracellular $\mathrm{Ca}^{2+}$ and mitochondrial homeostasis in the apoptosis of prostate cancer cells by deoxypodophyllotoxin. J Cell Biochem 114: 1124-1134, 2013.
27 Pan X, Zhang X, Sun H, Zhang J, Yan M and Zhang H: Autophagy inhibition promotes 5-fluorouraci-induced apoptosis by stimulating ROS formation in human non-small cell lung cancer A549 cells. PLoS One 8: e56679, 2013.

28 Maillet A, Yadav S, Loo YL, Sachaphibulkij K and Pervaiz S: A novel Osmium-based compound targets the mitochondria and triggers ROS-dependent apoptosis in colon carcinoma. Cell Death Dis 4: e653, 2013.

29 Shin S, Jing K, Jeong S, Kim N, Song KS, Heo JY, Park JH, Seo KS, Han J, Park JI, Kweon GR, Park SK, Wu T, Hwang BD and Lim K: The omega-3 polyunsaturated fatty acid DHA induces simultaneous apoptosis and autophagy via mitochondrial ROSmediated Akt-mTOR signaling in prostate cancer cells expressing mutant p53. Biomed Res Int 2013: 568671, 2013.

30 Chen Y, McMillan-Ward E, Kong J, Israels SJ and Gibson SB: Oxidative stress induces autophagic cell death independent of apoptosis in transformed and cancer cells. Cell Death Differ 15: 171-182, 2008. 